\title{
Local Shape Spectrum Analysis for 3D Facial Expression Recognition
}

\author{
Dmytro Derkach and Federico M. Sukno \\ Department of Information and Communication Technologies, Pompeu Fabra University, Barcelona, Spain
}

\begin{abstract}
We investigate the problem of facial expression recognition using 3D data. Building from one of the most successful frameworks for facial analysis using exclusively 3D geometry, we extend the analysis from a curve-based representation into a spectral representation, which allows a complete description of the underlying surface that can be further tuned to the desired level of detail. Spectral representations are based on the decomposition of the geometry in its spatial frequency components, much like a Fourier transform, which are related to intrinsic characteristics of the surface. In this work, we propose the use of Graph Laplacian Features (GLF), which results from the projection of local surface patches into a common basis obtained from the Graph Laplacian eigenspace. We test the proposed approach in the BU-3DFE database in terms of expressions and Action Units recognition. Our results confirm that the proposed GLF produces consistently higher recognition rates than the curves-based approach, thanks to a more complete description of the surface, while requiring a lower computational complexity. We also show that the GLF outperform the most popular alternative approach for spectral representation, Shape-DNA, which is based on the Laplace Beltrami Operator and cannot provide a stable basis that guarantee that the extracted signatures for the different patches are directly comparable.
\end{abstract}

\section{INTRODUCTION}

Human face plays an important role while expressing emotions such as happiness, satisfaction, surprise, fear, sadness or disgust. While there is consensus about the need to integrate multi-modal information for a complete understanding of human emotions, facial expressions are considered one of the most relevant channels for humans to regulate interactions both with the environment and with other persons [1].

During the past two decades, the problem of facial expression recognition has become very relevant. The growing interest in improving the interaction and cooperation between people and computers makes it necessary that automatic systems are able to react to a user and his emotions, as it takes place in natural human intercourse. Many applications such as virtual reality, video-conferencing, user profiling and customer satisfaction studies for broadcast and web services, require efficient facial expression recognition in order to achieve the desired results [2], [3]. Therefore, the impact of facial expression recognition on the above-mentioned application areas is constantly growing.

Methods for facial expression recognition are generally based on two possible imaging domains: 2D and 3D. Previous studies have focused primarily on the 2D domain (texture information) [4] due to the prevalence of data. With the rapid development of 3D imaging and scanning technologies, it becomes more and more popular using 3D face scans. Compared with 2D face images, 3D face scans contain detailed geometric shape information of facial surfaces, which remove the problems of illumination and pose variations that are inherent to the 2D modality. Thus, 3D-shape analysis has attracted increasing attention [5].

The availability of $3 \mathrm{D}$ information is not always fully exploited and, in many cases, 3D information is analyzed by directly applying 2D techniques to limited depth representation. This is typically done by using depth maps (2.5D representations), where the depth information is treated analogously to a gray-scale image and the 3D information is simply extracted by computing popular 2D texture descriptors such as LBPs [6], [7], [8] or Gabor filters [9], [10], [11]. Following a similar strategy, Zeng et al. [12] conformally mapped the $3 \mathrm{D}$ facial surface to a $2 \mathrm{D}$ unit disk and then considered it as a 2D image. More recently, deep convolutional neural networks has been explored in order to generate deep features [13] from this 2.5D representation.

However, in order to take full advantage of depth information we need approaches that are truly 3D. A notable approach in this direction, from Klassen et al., is based on the representation of surfaces with a finite number of level curves [14]. They showed that curves can be used to represent surface regions, being able to capture quite subtle deformations. Thus, 3D shape analysis can be performed by comparisons of corresponding level curves. It should be noted, however, that such comparison is not trivial, given that distances between 3D level curves should be computed based on the geodesic paths of their underlying manifold. An important step forward in this direction was presented by Srivastava et al. [15], who introduced a square-root velocity representation for analyzing curves in Euclidean spaces under a Riemannian metric. In particular, they computed geodesic paths between curves under this metric to obtain deformations between closed curves. Samir et al. [16] applied this curves-based approach for the analysis of facial surfaces. They represented a surface as an indexed collection of closed curves. These curves were extracted according to to their Euclidean distance from the tip of the nose, which is sensitive to deformations and, thus, can better capture differences related to variant expressions. Then, authors studied curves' differential geometry and endowed it with a Riemannian metric. In order to quantify differences between any two facial surfaces, the length of a geodesic was used. A similar framework was used in [17], [18] for analyzing 3D faces, with the goal of comparing, matching and averaging faces, with the difference that surfaces were represented by radial curves outflowing from the nose tip. Maalej et al. [19], based on an indexed collection of closed curves, emphasized the importance of using local regions instead of the entire face and proposed a local geometric analysis of the surface. 
They introduced a facial surfaces representation based on sets of level curves around landmarks. In their work, they used 70 landmarks and then extracted collections of closed curves using Euclidean distance. Thereby, 70 patches centered on the considered points represented the facial surface, where each patch consisted of an indexed collection of 3D closed curves. Further, they applied a Riemannian framework to derive $3 \mathrm{D}$ shape analysis and quantify similarity between corresponding patches on different 3D facial scans.

Despite the success of the level-curves framework, it could be argued that it is an incomplete representation of the 3D data, since it only captures part of the underlying surface, which is actually sampled by means of a finite number of curves. Spectral representations are based on the decomposition of the geometry in its (fundamental) frequency components, which are related to intrinsic characteristics of the surface, and correspond to the eigenvectors of the Laplace Beltrami Operator (LBO). The spectrum of the LBO is an isometric invariant, and it has been shown to be a powerful descriptor as a signature for (non-rigid) 3D shape matching and classification [20], [21]. The most popular of such descriptors was proposed by Reuter et al. [21], by taking the eigenvalues (i.e. spectrum) of its LaplaceBeltrami operator. Because such spectrum captures intrinsic shape information they called the method Shape-DNA. It was shown that this approach can be used (like DNA-test) to identify $3 \mathrm{D}$ objects or to detect similarities in practical applications. Several works used the Shape-DNA to identify objects for the purpose of copyright protection, but, to the best of our knowledge, it has not been applied for facial expression analysis.

\section{Contributions}

In this paper, we explore the use of spectral methods as local shape descriptors for 3D facial expression recognition. We show that the application of Shape-DNA is not the best way to deal with local face patches and that a fixedgraph basis, which we refer to as Graph Laplacian Features (GLF), provides superior results. This is theoretically sound given the impossibility to ensure a fixed ordering of the spectral components under the Shape-DNA approach [22]. Compared to the curves-based framework, the proposed method constitutes a generalization to a full representation of the surface patches resulting in higher accuracy and reduced computational complexity. We perform experiments over the BU-3DFE database and show that the proposed GLF approach consistently outperforms the curves-based and Shape-DNA alternatives, both in terms of expression and Action Unit recognition.

\section{SPECTRAL SHAPE ANALYSIS}

Spectral shape analysis relies on the decomposition of the surface geometry into its spatial frequency components (spectrum). Such representation allows to analyse the surface by examining the eigenvalues, eigenvectors or eigenspace projections of these fundamental frequencies.
One of the advantages of these methods is that they are invariant with respect to isometry, which means that these descriptors do not change with different isometric embeddings of the shape. In addition, their advantage is that they can be applied well to deformable objects. Spectral methods have been applied to solve a variety of problems including mesh compression, correspondence, smoothing, watermarking, segmentation, surface reconstruction etc. [23], [24], [25].

In our work, we use the spectrum based on Laplace operator for facial expression recognition. The Laplacians are the most commonly used operators for spectral mesh processing. As Chung stated in her book [26], results from spectral theory suggest that the Laplacian eigenvalues are tightly related to almost all major graph invariants. Thus, if data models the structures of a shape, either topology or geometry, then it is expected that its set of eigenvalues provides an appropriate characterization of the shape. The eigenvalues serve as compact global shape descriptor [25].

Several Laplacian operators have been proposed in the literature to compute the mesh spectrum. In this work we are especially interested in the two most popular ones:

1) Graph Laplacian, related to operators that have been widely studied in graph theory [26]. Despite this operator is based solely upon topological information, its eigenfunctions (i.e. eigenvectors) generally have a remarkable conformity to the mesh geometry [27]. On the other hand, the eigenfunctions of this operator are sensitive to aspects such as mesh resolution or triangulation.

2) Discretizations of the Laplace-Beltrami operator from Riemannian geometry [28], [29], which try to make basis dependant only on the underlying geometry and not its specific representation. This is the type of operator used in the Shape-DNA approach.

\section{A. Graph Laplacian}

Mesh (graph) Laplacian operators are linear operators that act on functions defined on the mesh and they depend purely on the mesh points (vertices) and their connectivity (e.g. triangulation). Thus, if mesh $M$ has $n$ vertices, a mesh Laplacian will be described by a $n \times n$ matrix $L$.

Given a mesh $M$ with vertices $V$ and edges $E, M=$ $(V, E)$, the graph Laplacian $L=L(M)$ is defined as

$$
L_{i j}= \begin{cases}-1 & \text { if }(i, j) \in E \\ d_{i} & \text { if } i=j \\ 0 & \text { otherwise }\end{cases}
$$

where $d_{i}$ is the degree or valence of vertex $i$.

Since this operator is determined purely by the connectivity of the mesh, it does not explicitly encode geometric information. However, as shown in the seminal work from Taubin [30], eigen-decomposition of the graph Laplacian produces an orthogonal basis whose components relate to spatial frequencies, much like a Fourier Transform. Projections of a mesh into the eigenspace of Laplacian operators have been proposed [31], [32] and used to derive shape 


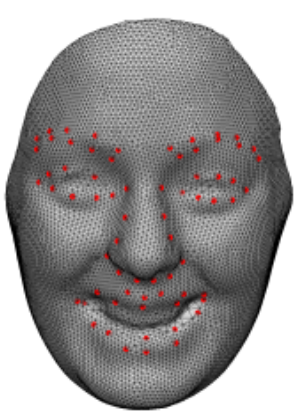

(a)

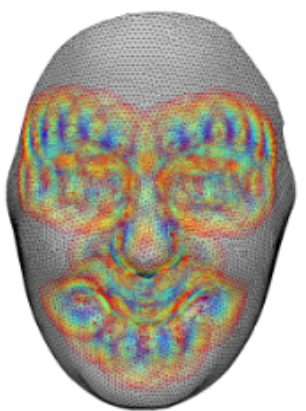

(b)

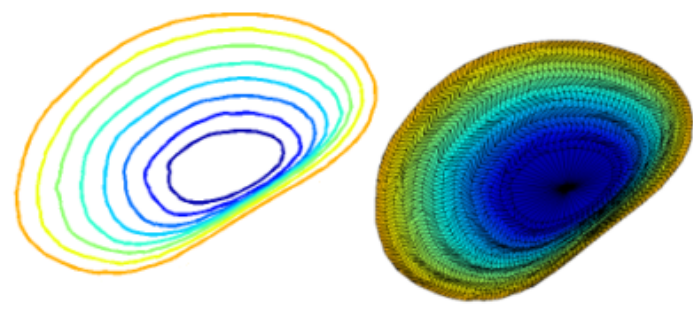

(c) (d)

Fig. 1. (a) 3D annotated facial shape model (68 landmarks); (b) closed curves extracted around the landmarks; (c) example of 8 level curves; (d) the mesh patche.

descriptors [33]. In face, eigenvectors are most frequently used to derive a spectral embedding of the input data (e.g. the mesh shape), since the spectral domain is more convenient to operate as it is low-dimensional and invariant to isometries while it still retains as much information about the input data as possible.

\section{B. Shape-DNA}

In Riemannian geometry, the Laplace operator can be generalized to operate on functions defined on a surface. In this case, the Laplace-Beltrami operator is of particular interest in geometry processing.

Ovsjanikov in [34] showed that the Laplace-Beltrami operator can be defined entirely in terms of the metric tensor on the manifold independently of the parametrization. Compared to the graph Laplacian, the Laplace-Beltrami operator does not operate on any mesh vertices, but rather on the underlying manifold itself. It depends continuously on the shape of the surface [35].

The Laplace operator based on the cotan formula represents the most popular discrete approximation to the LaplaceBeltrami operator currently used for geometry processing. This operator can be presented as a product of a diagonal and symmetric matrix $L=B^{-1} S$. Where $B^{-1}$ is a diagonal matrix whose diagonal entries are Voronoi areas [36] for all vertices and $S$ is a symmetric matrix defined [37]:

$$
S_{i j}= \begin{cases}-w_{i j} & \text { if }(i, j) \in E \\ \sum_{k \in N(i)} w_{i k} & \text { if } i=j \\ 0 & \text { otherwise }\end{cases}
$$

where $w_{i j}=\left(\cot \alpha_{i j}+\cot \beta_{i j}\right), \alpha_{i j}$ and $\beta_{i j}$ are the angles opposite if the edge $(i, j)$ (Fig. 2). $N(i)$ is a set of vertices that are adjacent to vertixe $i$.

A significant amount of geometric and topological information is known to be contained in the spectrum. Since the spectrum (i.e. the eigenvalues) of the LaplaceBeltrami operator contains intrinsic shape information Reuter et al proposed to use them as shape signature or Shape-DNA [21]. Shape-DNA can be used to identify shapes and detect similarities.

In order to extract appropriate eigenvalues, matrix $L$ should be symmetric. The main advantage offered by sym- metric matrices is that they possess real eigenvalues whose eigenvectors form an orthogonal basis [38]. Although L itself is not symmetric in general, it is similar to the symmetric matrix $O=B^{-1 / 2} S B^{-1 / 2}$ since

$$
L=B^{-1} S=B^{-1 / 2} B^{-1 / 2} S B^{-1 / 2} B^{1 / 2}=B^{-1 / 2} O B^{1 / 2}
$$

Thus, $L$ and $O$ have the same real eigenvalues [25]. Further, these eigenvalues can be compared for shape identification.

\section{SPECTRAL REPRESENTATION OF FACIAL PATCHES}

In order to explore the use of spectral methods as local shape descriptors, we represented a surface based on surface patches. For this purpose, we choose to consider $N$ reference points (landmarks) $\left\{r_{l}\right\}_{1 \leq l \leq N}$ (Fig. 1(a)) and, following [19], their associated sets of level curves $\left\{c_{\lambda}^{l}\right\}_{\lambda_{\min } \leq \lambda \leq \lambda_{\max }}$ (Fig. 1(b)). These curves were extracted over the surface $S$ centered at the considered landmark points, where $\lambda$ is the distance between the reference point $r_{l}$ and the point belonging to the curve $c_{\lambda}^{l}, \lambda_{\min }$ and $\lambda_{\max }$ stand for the minimum and maximum values taken by $\lambda$. The computation of the curves was performed using an Euclidean distance function:

$$
c_{\lambda}^{l}=\left\{p \in S \mid\left\|r_{l}-p\right\|=\lambda\right\} \subset S, \lambda \in\left[\lambda_{\min }, \lambda_{\max }\right]
$$

In that way, $c_{\lambda}^{l}$ is a curve, which consists of a collection of points $\{p\}$ located at an equal distance $\lambda$ from point $r_{l}$. Accordingly, each facial surface is represented by patches that consist of sets of level curves around landmarks.

Once the patches are extracted, we aim to study their shape. Because we want to calculate the mesh spectra for the

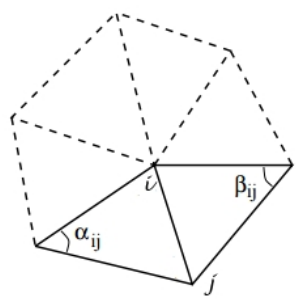

Fig. 2. 1-ring neighbors and angles opposite to an edge. 


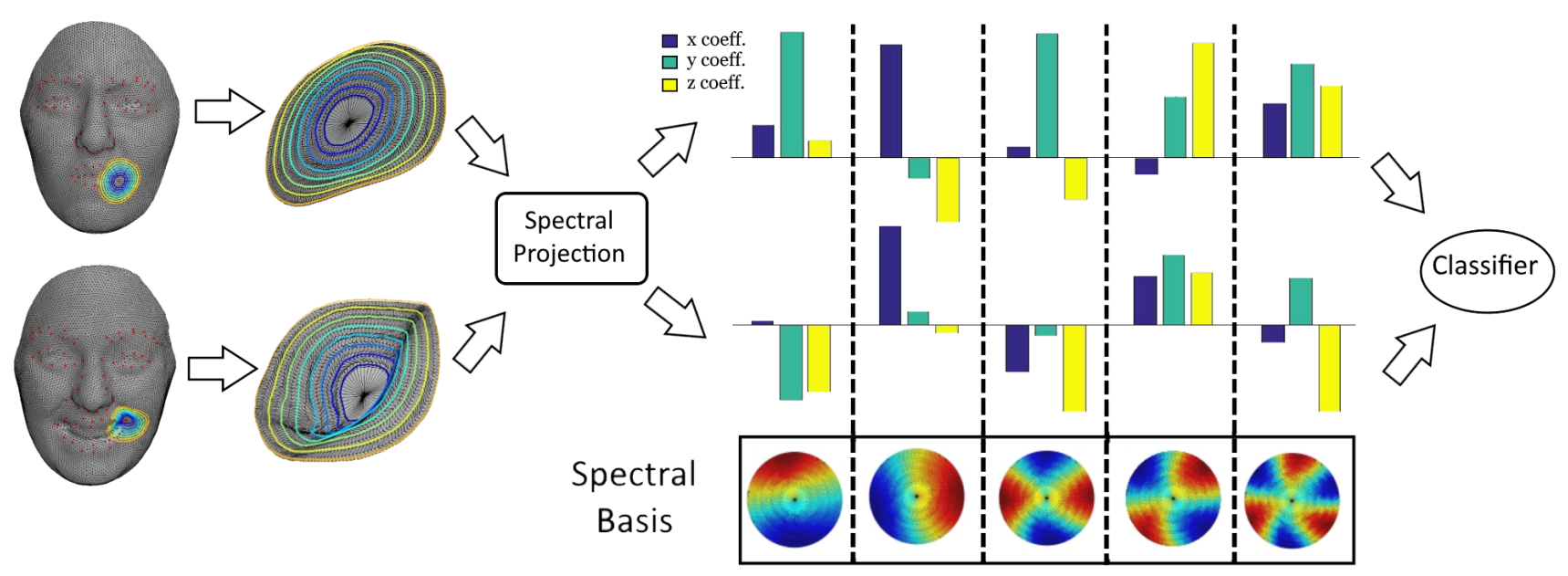

Fig. 3. Schematic representation of the proposed approach. For each facial landmark, a surface patch is extracted to describe its local geometry. Each patch is projected into a common eigenspace to obtain a set of spectral coefficients that constitute our features. The eigenspace is computed off-line as the spectrum of the Graph Laplacian operator which depends exclusively on the connectivity of vertices and is therefore common for all patches. The spectral coefficients can be interpreted as loadings that weight the contribution of the spectral components. In the figure we display the coefficients of the first 5 spectral components, as well as the spatial patterns produced by their corresponding eigenvectors.

patches, we need to convert level curves to surface patches. Notice that, conceptually, we may directly extract the patches with no need to first extract the curves, but proceeding this way facilitates comparison to [19] and, as we explain below, allows for using directly the graph Laplacian instead of the Laplace-Beltrami operator. To generate the mesh patches we re-sample the curves uniformly (as done in [19]) and define a unique connectivity between them, which will be shared by all patches (Fig. 1(d)).

After these pre-processing steps, we extract spectral features for facial expression analysis. We propose to do so using the Graph Laplacian, since this is the more theoretically sound approach under our settings. We also compare the results obtained by Shape-DNA, arguably the most widespread method to extract spectral features from 3D meshes. Specifically, spectral features are extracted as follows:

- Graph Laplacian: Whereas graph Laplacian depends only on the connectivity between vertices, we calculated matrix $L$ using formula (1) only once. Eigenvalues and eigenvectors were obtained from this matrix. Because we generated all our mesh patches with the same order of connectivity, the set of eigenvectors constitutes a common basis to represent the spatial spectrum of all patches. Therefore, we used these eigenvectors to project mesh coordinates into the common eigenspace. These projections constitute our feature vectors, and are directly comparable between patches.

- Shape-DNA: The second type of features was obtained using the Laplace-Beltrami operator (2). This operator was calculated separately on each mesh-patch, because it depends not only on the connectivity but also on the location of the vertices. Thus, the eigen-decomposition of each patch produces a different eigenspace, which is tuned to the geometry of that specific patch. Projections into the eigenspace are therefore no longer comparable, but the eigenvalues resulting from each decomposition have been proven discriminative [23], hence we use them as feature vectors.

To drive the classification experiments, we employed two different classifiers - support vector machines (SVM) invoking the LIBSVM software [39] and Fisher's Linear Discriminant Analysis (FLDA) [40]. A schematic diagram of the proposed framework is presented in Fig. 3.

\section{EXPERIMENTS}

In the following section, we provide the details of our experiments on feature extraction with the proposed spectral analysis for facial expression recognition.

\section{A. Experimental setting}

In order to evaluate the proposed local shape spectrum analysis, we use the BU-3DFE database [41], which is one of the most widely used corpora for facial expression analysis in 3D. This database consist of 3D face scans of 100 subjects with different facial expressions. There are also variations in race, gender and age. Scans are annotated according to the six prototypical facial expressions (anger, disgust, fear, happiness, sadness and surprise) at four different intensity levels. For our experiments, we have used the scans from all 100 subjects at the two highest intensity levels. Thus, our dataset consists of 1200 3D face scans, namely two intensity levels for each of the six facial expressions from 100 subjects.

Accompanying each facial scan there are 83 manually labeled landmarks. From these, 15 landmarks correspond to the silhouette contour and have arguably little validity in a 3D setting, hence we considered the subset of $N=68$ landmarks laying within the face area. All facial scans were represented by 68 patches $\left\{c_{\lambda}^{l}\right\}_{\lambda_{\min } \leq \lambda \leq \lambda_{\max }}$, Where, each patch consist of 15 level curves (Fig. 1(c)) $\left(\lambda_{\min }=5, \lambda_{\max }=20\right)$ and 
TABLE I

AVERAGE ACCURACY OF THE THREE METHODS USING TWO CLASSIFIERS

\begin{tabular}{c||ccc}
\hline & Curves & Graph Laplacian & Shape-DNA \\
\hline \hline FLDA & $77.53 \%$ & $81 \%$ & $73.5 \%$ \\
SVM & $78.2 \%$ & $81.5 \%$ & $73.62 \%$ \\
\hline
\end{tabular}

TABLE II

AVERAGE CONFUSION MATRIX OF GRAPH LAPLACIAN USING 50 EIGENVALUES AND AN SVM CLASSIFIER

\begin{tabular}{c||cccccc}
\hline$\%$ & AN & DI & FE & HA & SA & SU \\
\hline \hline AN & $\mathbf{8 5 . 5 8}$ & 4.14 & 1.26 & 0.5 & 8.52 & 0 \\
DI & 7.5 & $\mathbf{7 5 . 3 1}$ & 8.76 & 3.01 & 0.9 & 4.52 \\
FE & 5.58 & 8.6 & $\mathbf{6 5 . 1 2}$ & 12.55 & 2.59 & 5.56 \\
HA & 0 & 2.16 & 6.87 & $\mathbf{8 9 . 5}$ & 0 & 0.9 \\
SA & 14.5 & 0.76 & 7.46 & 0 & $\mathbf{7 7 . 2}$ & 0 \\
SU & 0 & 1.72 & 3.53 & 1.2 & 0 & $\mathbf{9 3 . 5}$ \\
\hline
\end{tabular}

each curve is a collection of points situated at an equal distance from the considered landmarks.

The dataset was arbitrarily divided into ten identitydisjoint sets; each of these (composed on 120 samples) was tested with models trained from the remaining nine sets (1080 samples). Thus, the recognition rates are obtained by averaging the results over the 10 sets (10-fold crossvalidation).

\section{B. Results on Expression Recognition}

Our first experiment consists on a direct comparison of the proposed spectral features (based on GLF) with respect to the curves-framework and with respect to Shape-DNA, which constitute the straight-forward spectral alternative. This was done in the context of expression recognitions targeting the six basic emotions. (anger (AN), disgust (DI), fear (FE), happiness (HA), sadness (SA), surprise (SU)). Table I summarizes the average accuracy obtained by each approach. It can be seen that the spectral features based on the Graph Laplacian outperform the curves-based approach, which suggest that they can capture a more complete information about the facial patches. It is also interesting to see that ShapeDNA features obtain the lowest accuracy among the three methods. This confirms the theoretical limitations already highlighted with respect to the direct application of ShapeDNA to surface patches: given two shapes to compare under a spectral representation, small differences between them can modify the eigen-decomposition to the extent that the eigenvalues change their relative order producing a swapping of the extracted basis [22]. Such swaps make the direct comparison of eigenvalues used in Shape-DNA conceptually incorrect. Fixing this would require matching algorithms to appropriately re-order the resulting eigenvalues. Our GLF do not suffer from this issue as they result from a projection into a common basis, which only depends on the connectivity of the patches.

To put our results in a wider context, we can also compare them to other methods reporting expression recognition rates
TABLE III

AVERAGE CONFUSION MATRIX OF SHAPE-DNA USING 50 EIGENVALUES AND AN SVM CLASSIFIER

\begin{tabular}{c||cccccc}
\hline$\%$ & AN & DI & FE & HA & SA & SU \\
\hline \hline AN & $\mathbf{7 7 . 2 1}$ & 5.87 & 2.71 & 1.21 & 12.98 & 0 \\
DI & 7.45 & $\mathbf{7 5 . 5 3}$ & 7.98 & 3.87 & 1.52 & 3.61 \\
FE & 7.23 & 9.82 & $\mathbf{5 2 . 5 3}$ & 15.46 & 9.56 & 5.37 \\
HA & 2.1 & 3.45 & 12.5 & $\mathbf{8 0 . 4 9}$ & 0 & 1.46 \\
SA & 19.76 & 3.51 & 6.32 & 0.31 & $\mathbf{6 9 . 5 1}$ & 0.57 \\
SU & 0.49 & 1.52 & 10.23 & 1.54 & 0.49 & $\mathbf{8 5 . 7 4}$ \\
\hline
\end{tabular}

TABLE IV

AVERAGE CONFUSION MATRIX OF APPROACH BASED ON "DISTANCES BETWEEN CURVES" USING AN SVM CLASSIFIER

\begin{tabular}{c||cccccc}
\hline$\%$ & AN & DI & FE & HA & SA & SU \\
\hline \hline AN & $\mathbf{7 8 . 9 6}$ & 6.08 & 3.83 & 0.55 & 10.56 & 0 \\
DI & 5.49 & $\mathbf{7 6 . 1 4}$ & 5.09 & 4.42 & 2.87 & 5.97 \\
FE & 3.92 & 7.08 & $\mathbf{6 3 . 4 5}$ & 13.24 & 6.12 & 6.17 \\
HA & 1.11 & 2.78 & 9.45 & $\mathbf{8 6 . 6 5}$ & 0 & 0 \\
SA & 12.26 & 0.58 & 8.28 & 0 & $\mathbf{7 8 . 8 6}$ & 0 \\
SU & 0 & 2.78 & 8.86 & 1.08 & 0.55 & $\mathbf{8 6 . 7 1}$ \\
\hline
\end{tabular}

on the BU3DFE database. As detailed in [12], only methods whose experimental settings consider the whole set of 100 subjects are fairly comparable. Among these, expression recognition rates vary between $68.2 \%$ [12] and $82.7 \%$ [42], while our average recognition rates reach $81.5 \%$. Notice that in our case we use a single type of feature (GLF), while most other works achieving high recognition rates use combinations of multiple features.

To provide a more extensive review of our results, Tables II, III and IV show the average confusion matrices for each of the approaches using SVM classifier. It can be seen that among the six basic expressions surprise, happiness and anger were recognized the best. In contrast, fear and disgust were the most difficult expressions to predict. We also observe that GLF consistently outperform both the curvebased and Shape-DNA approaches for most expressions, with the only major exception being Disgust, where it performs similarly but slightly worse than the competing alternatives.

An important factor when using spectral decomposition methods is the number of considered components. All results reported above correspond to the first 50 components (eigenvalues in the case of Shape-DNA, projections in the eigenspace in the case of GLF). We also repeated the expression recognition experiments for different numbers of components and found the performance of both GLF and Shape-DNA to be relatively as long as at least 10 components were used (see Table V). Tests extended only up to 200 components, since increasing the components implies also more computational load while not bringing improvements in accuracy.

\section{Action Unit Estimation}

Since our approach is based on the aggregation of localized descriptors of the facial surface, it would make sense that it can also be applied to the estimation of Action Units 
TABLE V

AVERAGE ACCURACY OF FACIAL EXPRESSION RECOGNITION UNDER DIFFERENT CLASSIFIERS FOR DIFFERENT NUMBERS OF EIGENVALUES

\begin{tabular}{|c|c|c|c|c|c|c|c|c|c|c|}
\hline Features & \multicolumn{5}{|c|}{ Graph Laplacian } & \multicolumn{5}{|c|}{ Shape-DNA } \\
\hline Eigenvalues & 200 & 100 & 50 & 30 & 10 & 200 & 100 & 50 & 30 & 10 \\
\hline FLDA & $80.25 \%$ & $79.92 \%$ & $81 \%$ & $80.25 \%$ & $79.42 \%$ & $71.17 \%$ & $71.25 \%$ & $73.5 \%$ & $72.83 \%$ & $71.08 \%$ \\
\hline SVM & $80.3 \%$ & $80 \%$ & $81.5 \%$ & $79.5 \%$ & $80.83 \%$ & $71.2 \%$ & $71.33 \%$ & $73.62 \%$ & $72.9 \%$ & $71 \%$ \\
\hline
\end{tabular}

(AU). AUs are designed to capture any anatomically feasible facial deformation [43], thereby combinations of AUs can be used to describe any of the six basic expressions [44], as well as any other anatomically feasible facial expression. Each of the expressions in the BU-3DFE database was manually annotated with corresponding sets of AUs by two coders ${ }^{1}$. The resulting annotations were checked for consistency of the obtained AU frequencies per expression and co-occurrences of AUs with [45], [46], [47]. Then, experiments on AUs recognition were performed under the same conditions as the expressions recognition tests.

Table VI shows the weighted average F1-score for each AU (weighted proportionally to the number of samples per $\mathrm{AU})$. One common characteristic of all the approaches is that they all recognized AU25, AU26 better that any other. Also, analysing the table, we can see that detection of AU1, AU2, AU4, AU5 and AU12 can be said reliable. The worst detected AU was AU15.

When comparing among features, our results show the same tendency observed in the expression recognition experiments. The best performance was obtained by GLF, which clearly outperformed Shape-DNA and was also slightly better than the approach based on geodesic distance between curves. Regarding the latter, while the average recognition accuracy of GLF and curves were rather similar, it should be noted that GLF consistently outperformed curves in 15 out of the 17 tested AUs.

\section{CONCLUSIONS}

In this work, we extend the analysis of 3D geometry from a curve-based representation into a spectral representation. This representation allows to build a complete description of the underlying surface that can be further tuned to the desired level of detail. We propose the use of Graph Laplacian Features (GLF), which result from the projection of local surface patches into a common basis obtained from the Graph Laplacian eigenspace, much like a Fourier transform into the spatial frequency bassis of the surface patches. Further, we compare our approach with two others approaches. The first one is the curves-based framework and the second one is the straight-forward alternative for spectral representation, Shape-DNA, which is based on the Laplace Beltrami Operator. We show that the straight-forward application of ShapeDNA is not the best way to deal with local face patches, since it cannot provide a stable basis to guarantee that the extracted signatures for the different patches are directly comparable.

We tested the proposed approach in the BU-3DFE database in terms of expressions and Action Units recog-

\footnotetext{
${ }^{1}$ Available at http://fsukno.atspace.eu/Research.htm\#FG2017a
}

TABLE VI

AVERAGE F1-SCORE RECOGNITION RESULTS OF AUS USING 50 EIGENVALUES

\begin{tabular}{c||c||ccc}
\hline AU & \# Samples & Curves & Graph Laplacian & Shape-DNA \\
\hline \hline 1 & 333 & 0.74 & 0.75 & 0.73 \\
2 & 302 & 0.77 & 0.78 & 0.73 \\
4 & 423 & 0.77 & 0.79 & 0.74 \\
5 & 304 & 0.76 & 0.80 & 0.71 \\
6 & 68 & 0.42 & 0.46 & 0.45 \\
7 & 370 & 0.69 & 0.73 & 0.63 \\
9 & 99 & 0.55 & 0.56 & 0.47 \\
10 & 136 & 0.64 & 0.67 & 0.57 \\
12 & 177 & 0.74 & 0.76 & 0.70 \\
15 & 69 & 0.37 & 0.34 & 0.30 \\
16 & 122 & 0.50 & 0.52 & 0.39 \\
17 & 130 & 0.48 & 0.50 & 0.42 \\
20 & 84 & 0.28 & 0.3 & 0.25 \\
23 & 134 & 0.42 & 0.50 & 0.38 \\
24 & 125 & 0.57 & 0.61 & 0.62 \\
25 & 709 & 0.94 & 0.94 & 0.92 \\
26 & 230 & 0.85 & 0.88 & 0.86 \\
\hline Avrg & Total: 3815 & $\mathbf{0 . 7 2}$ & $\mathbf{0 . 7 4}$ & $\mathbf{0 . 6 9}$ \\
\hline
\end{tabular}

nition. Our results show that the proposed GLF consistently outperform the curves-based and Shape-DNA alternatives, both in terms of expression recognition and Action Unit recognition. Moreover, the recognition rates of shape-DNA are even lower than the curves-based framework, as predicted by the theory: in spite of upgrading the curves-based representation to a full-surface description, similarly to GLF, the instabilities of the bases extracted by Shape-DNA result in a decreased performance.

Interestingly, the accuracy improvement brought by GLF is obtained also at a lower computational cost. Considering the extraction of patches as a common step between the three compared approaches, the curve-based framework requires a costly elastic deformation between corresponding curves (e.g. based on splines) and Shape-DNA requires computing the eigen-decomposition of each new patch to be analyzed. In contrast, GLF only require the projection of the patch geometry into the Graph Laplacian eigenspace, which is common to all patches and can thus be pre-computed offline.

\section{ACKNOWLEDGMENTS}

This work is partly supported by the Spanish Ministry of Economy and Competitiveness under the Ramon y Cajal fellowships and the Maria de Maeztu Units of Excellence Programme (MDM-2015-0502). 


\section{REFERENCES}

[1] M. Pantic, "Machine analysis of facial behaviour: Naturalistic and dynamic behaviour," Philosophical Transactions of the Royal Society B: Biological Sciences, vol. 364, no. 1535, pp. 3505-3513, 2009.

[2] J. M. Girard, J. F. Cohn et al., "Social risk and depression: Evidence from manual and automatic facial expression analysis," in $F G$, International Conference and Workshops on. IEEE, 2013, pp. 1-8.

[3] D. McDuff, R. El Kaliouby et al., "Predicting online media effectiveness based on smile responses gathered over the internet," in Automatic Face and Gesture Recognition, International Conference and Workshops on. IEEE, 2013, pp. 1-7.

[4] G. Sandbach, S. Zafeiriou et al., "Static and dynamic 3d facial expression recognition: A comprehensive survey," Image and Vision Computing, vol. 30, no. 10, pp. 683-697, 2012.

[5] H. Li, H. Ding et al., "An efficient multimodal $2 d+3 d$ feature-based approach to automatic facial expression recognition," Computer Vision and Image Understanding, vol. 140, pp. 83-92, 2015.

[6] Z. Guo, Y. Zhang et al., "A method based on geometric invariant feature for 3d face recognition," in Image and Graphics, Fifth International Conference on. IEEE, 2009, pp. 902-906.

[7] Y. Wang and M. Meng, "3d facial expression recognition on curvature local binary patterns," in Intelligent Human-Machine Systems and Cybernetics, International Conference on, vol. 2. IEEE, 2013, pp. $123-126$.

[8] W. Wang, W. Zheng, and Y. Ma, "3d facial expression recognition based on combination of local features and globe information," in Intelligent Human-Machine Systems and Cybernetics, Sixth International Conference on, vol. 2. IEEE, 2014, pp. 20-25.

[9] T. Yun and L. Guan, "Human emotion recognition using real 3d visual features from gabor library," in Multimedia Signal Processing, International Workshop on. IEEE, 2010, pp. 505-510.

[10] S. Xie, S. Shan et al., "Fusing local patterns of gabor magnitude and phase for face recognition," Transactions on Image Processing, vol. 19, no. 5, pp. 1349-1361, 2010.

[11] J. D'Hose, J. Colineau et al., "Precise localization of landmarks on 3d faces using gabor wavelets," in Biometrics: Theory, Applications, and Systems. First International Conference on. IEEE, 2007, pp. 1-6.

[12] W. Zeng, H. Li et al., "An automatic 3d expression recognition framework based on sparse representation of conformal images," in Automatic Face and Gesture Recognition, International Conference and Workshops on. IEEE, 2013, pp. 1-8.

[13] H. Li, J. Sun et al., "Deep representation of facial geometric and photometric attributes for automatic $3 \mathrm{~d}$ facial expression recognition," arXiv preprint arXiv:1511.03015, 2015.

[14] E. Klassen and A. Srivastava, "Geodesics between $3 \mathrm{~d}$ closed curves using path-straightening," in European conference on computer vision. Springer, 2006, pp. 95-106.

[15] A. Srivastava, E. Klassen et al., "Shape analysis of elastic curves in euclidean spaces," IEEE Transactions on Pattern Analysis and Machine Intelligence, vol. 33, no. 7, pp. 1415-1428, 2011.

[16] C. Samir, A. Srivastava et al., "An intrinsic framework for analysis of facial surfaces," International Journal of Computer Vision, vol. 82, no. 1, pp. 80-95, 2009.

[17] H. Drira, B. B. Amor et al., "3d face recognition under expressions, occlusions, and pose variations," IEEE Transactions on Pattern Analysis and Machine Intelligence, vol. 35, no. 9, pp. 2270-2283, 2013.

[18] B. B. Amor, H. Drira et al., "4-d facial expression recognition by learning geometric deformations," IEEE transactions on cybernetics, vol. 44, no. 12, pp. 2443-2457, 2014.

[19] A. Maalej, B. B. Amor et al., "Shape analysis of local facial patches for 3d facial expression recognition," Pattern Recognition, vol. 44, no. 8, pp. 1581-1589, 2011.

[20] Z. Karni and C. Gotsman, "Spectral compression of mesh geometry," in Proceedings of the 27th annual conference on Computer graphics and interactive techniques. ACM Press/Addison-Wesley Publishing Co., 2000, pp. 279-286.

[21] M. Reuter, F.-E. Wolter, and N. Peinecke, "Laplace-beltrami spectra as shape-dnaof surfaces and solids," Computer-Aided Design, vol. 38, no. 4, pp. 342-366, 2006

[22] V. Jain, H. Zhang, and O. van Kaick, "Non-rigid spectral correspondence of triangle meshes," International Journal of Shape Modeling, vol. 13, no. 01, pp. 101-124, 2007.

[23] M. Reuter, "Hierarchical shape segmentation and registration via topological features of laplace-beltrami eigenfunctions," International Journal of Computer Vision, vol. 89, no. 2-3, pp. 287-308, 2010.
[24] A. Nealen, T. Igarashi et al., "Laplacian mesh optimization," in Proceedings of the 4th international conference on Computer graphics and interactive techniques in Australasia and Southeast Asia. ACM, 2006, pp. 381-389.

[25] H. Zhang, O. Van Kaick, and R. Dyer, "Spectral mesh processing," in Computer graphics forum, vol. 29, no. 6. Wiley Online Library, 2010, pp. 1865-1894.

[26] F. R. Chung, Spectral graph theory. American Mathematical Soc., 1997, vol. 92

[27] M. Isenburg, S. Gumhold, and C. Gotsman, "Connectivity shapes," in Proceedings of the conference on Visualization'01. IEEE Computer Society, 2001, pp. 135-142.

[28] I. Chavel, Eigenvalues in Riemannian geometry. Academic press, 1984, vol. 115.

[29] S. Rosenberg, The Laplacian on a Riemannian manifold: an introduction to analysis on manifolds. Cambridge University Press, 1997, no. 31 .

[30] G. Taubin, "A signal processing approach to fair surface design," in Proceedings of the 22nd annual conference on Computer graphics and interactive techniques. ACM, 1995, pp. 351-358.

[31] M. Desbrun, M. Meyer et al., "Implicit fairing of irregular meshes using diffusion and curvature flow," in Proceedings of the 26th annual conference on Computer graphics and interactive techniques. ACM Press/Addison-Wesley Publishing Co., 1999, pp. 317-324.

[32] B. Kim and J. Rossignac, "Geofilter: Geometric selection of mesh filter parameters," in Computer Graphics Forum, vol. 24, no. 3. Wiley Online Library, 2005, pp. 295-302.

[33] C. T. Zahn and R. Z. Roskies, "Fourier descriptors for plane closed curves," IEEE Transactions on computers, vol. 100, no. 3, pp. 269 281, 1972.

[34] M. Ovsjanikov, J. Sun, and L. Guibas, "Global intrinsic symmetries of shapes," in Computer graphics forum, vol. 27, no. 5. Wiley Online Library, 2008, pp. 1341-1348.

[35] R. Courant and D. Hilbert, Methods of mathematical physics. CUP Archive, 1965, vol. 1.

[36] M. Meyer, M. Desbrun et al., "Discrete differential-geometry operators for triangulated 2-manifolds," in Visualization and mathematics III. Springer, 2003, pp. 35-57.

[37] H. Wang, Z. Su et al., "Empirical mode decomposition on surfaces," Graphical Models, vol. 74, no. 4, pp. 173-183, 2012.

[38] R. Bhatia, Matrix analysis. Springer Science \& Business Media, 2013, vol. 169.

[39] C.-C. Chang and C.-J. Lin, "Libsvm: a library for support vector machines," ACM Transactions on Intelligent Systems and Technology, vol. 2, no. 3, p. 27, 2011.

[40] M. Welling, "Fisher linear discriminant analysis," Department of Computer Science, University of Toronto, vol. 3, pp. 1-4, 2005.

[41] L. Yin, X. Wei et al., "A 3d facial expression database for facial behavior research," in international conference on automatic face and gesture recognition. IEEE, 2006, pp. 211-216.

[42] X. Yang, D. Huang et al., "Automatic 3d facial expression recognition using geometric scattering representation," in Automatic Face and Gesture Recognition, International Conference and Workshops on, vol. 1. IEEE, 2015, pp. 1-6.

[43] P. Ekman, "Strong evidence for universals in facial expressions: a reply to russell's mistaken critique." 1994.

[44] A. Ruiz, J. Van de Weijer, and X. Binefa, "From emotions to action units with hidden and semi-hidden-task learning," in Proceedings of the IEEE International Conference on Computer Vision, 2015, pp. 3703-3711.

[45] S. Du, Y. Tao, and A. M. Martinez, "Compound facial expressions of emotion," Proceedings of the National Academy of Sciences, vol. 111, no. 15, pp. E1454-E1462, 2014.

[46] J. Wang, S. Wang et al., "Capture expression-dependent au relations for expression recognition," in Multimedia and Expo Workshops, International Conference on. IEEE, 2014, pp. 1-6.

[47] K. Zhao, W.-S. Chu et al., "Joint patch and multi-label learning for facial action unit detection," in Proceedings of the IEEE Conference on Computer Vision and Pattern Recognition, 2015, pp. 2207-2216. 